MATHEMATICS OF COMPUTATION

Volume 75, Number 254, Pages 631-640

S 0025-5718(05)01866-1

Article electronically published on December 19, 2005

\title{
RUNGE-KUTTA TIME DISCRETIZATIONS OF NONLINEAR DISSIPATIVE EVOLUTION EQUATIONS
}

\author{
ESKIL HANSEN
}

\begin{abstract}
Global error bounds are derived for Runge-Kutta time discretizations of fully nonlinear evolution equations governed by $m$-dissipative vector fields on Hilbert spaces. In contrast to earlier studies, the analysis presented here is not based on linearization procedures, but on the fully nonlinear framework of logarithmic Lipschitz constants in order to extend the classical $B$-convergence theory to infinite-dimensional spaces. An algebraically stable Runge-Kutta method with stage order $q$ is derived to have a global error which is at least of order $q-1$ or $q$, depending on the monotonicity properties of the method.
\end{abstract}

\section{INTRODUCTION}

The aim of this paper is to analyze Runge-Kutta time discretizations of the evolution equation

$$
\dot{u}=f(u), \quad u(0)=\eta
$$

where $u:[0, \infty) \rightarrow X$ and the vector field $f$ is a nonlinear $m$-dissipative map [1] on the real-valued Hilbert space $X$. Such vector fields are found in a wide range of applications, e.g., advection-diffusion-reaction processes. Multistep time discretizations of these evolution equations have been treated in (7), and a similar approach is taken here when analyzing Runge-Kutta approximations. Earlier studies of time discretizations on infinite-dimensional spaces have predominantly considered semilinear or quasi-linear vector fields; see, e.g., 9, 10, 16. It is not until recently that the fully nonlinear setting has been addressed [5, 11, 12. Here, it is assumed that the linearization of the vector field is a sectorial map, which is not generally true for $m$-dissipative maps. Hence a different approach is needed. Our idea is to generalize the classical $B$-convergence theory [3, 4, 6, for Runge-Kutta approximations of ordinary differential equations to approximations of evolution equations on infinite-dimensional spaces. This is done by extending the two fundamental analytic tools of the theory, namely the logarithmic norm of matrices and the monotonicity condition

$$
\langle u-v, f(u)-f(v)\rangle \leq 0
$$

These tools are merely special cases of the so-called logarithmic Lipschitz constants which are well defined on infinite-dimensional spaces and therefore enable us to mimic the proofs of the $B$-convergence theory in the present context.

Received by the editor December 14, 2004.

2000 Mathematics Subject Classification. Primary 65J15, 65M12.

Key words and phrases. Nonlinear evolution equations, logarithmic Lipschitz constants, $m$ dissipative maps, Runge-Kutta methods, algebraic stability, $B$-convergence.

(C)2005 American Mathematical Society Reverts to public domain 28 years from publication 


\section{Preliminaries}

This paper is based on the theory of logarithmic Lipschitz constants which was developed in [8, 14, 15. A short summary of the theory follows below. Assume that $X$ is a real-valued Hilbert space equipped with the inner product $\langle\cdot, \cdot\rangle_{X}$ and the corresponding norm $\|\cdot\|_{X}$. The map $f$ is a nonlinear map on $X$ with domain $D(f)$ and range $R(f)$. The Lipschitz constants of $f$ on $X$ are defined as follows.

Definition 2.1. For arbitrary $u, v \in D(f)$ define the $l u b$ and glb Lipschitz constants of $f$ on $X$ by

$$
L_{X}[f]:=\sup _{u \neq v} \frac{\|f(u)-f(v)\|_{X}}{\|u-v\|_{X}}, \quad l_{X}[f]:=\inf _{u \neq v} \frac{\|f(u)-f(v)\|_{X}}{\|u-v\|_{X}} .
$$

The basic properties of the Lipschitz constants are:

Proposition 2.2. Assume that $D(f) \bigcap D(g) \neq \emptyset$ in property $(3)$ and $R(g) \subseteq D(f)$ in property (4). Then

(1) $L_{X}[f] \geq 0$

(2) $L_{X}[\alpha f]=|\alpha| L_{X}[f]$,

(3) $L_{X}[f+g] \leq L_{X}[f]+L_{X}[g]$,

(4) $L_{X}[f \circ g] \leq L_{X}[f] L_{X}[g]$.

Next, the logarithmic Lipschitz constants are introduced.

Definition 2.3. For arbitrary $u, v \in D(f)$, define the $l u b$ and $g l b$ logarithmic Lipschitz constants of $f$ on $X$ as

$$
M_{X}[f]:=\sup _{u \neq v} \frac{\langle u-v, f(u)-f(v)\rangle_{X}}{\|u-v\|_{X}^{2}}, \quad m_{X}[f]:=\inf _{u \neq v} \frac{\langle u-v, f(u)-f(v)\rangle_{X}}{\|u-v\|_{X}^{2}} .
$$

Some of the basic properties of the logarithmic Lipschitz constants are:

Proposition 2.4. Assume that $D(f) \bigcap D(g) \neq \emptyset$ in property (4). Then

(1) $m_{X}[-f]=-M_{X}[f]$,

(2) $-L_{X}[f] \leq m_{X}[f] \leq l_{X}[f]$,

(3) $m_{X}[\alpha f]=\alpha m_{X}[f], \quad \alpha \geq 0$,

(4) $m_{X}[f]+m_{X}[g] \leq m_{X}[f+g]$.

Observe that the second property of Proposition 2.4 is a direct consequence of the Cauchy-Schwarz inequalities.

Lemma 2.5. If $l_{X}[f]>0$, then $f$ is injective and $L_{X}\left[f^{-1}\right]=l_{X}[f]^{-1}$.

Proof. Definition 2.1 trivially yields that $f$ is injective when $l_{X}[f]>0$, which implies that $f^{-1}: R(f) \rightarrow D(f)$ is well defined. For every $u_{1}, u_{2} \in D(f)$ let $v_{1}:=f\left(u_{1}\right), v_{2}:=f\left(u_{2}\right)$. Then

$$
l_{X}[f]^{-1}=\sup _{u_{1} \neq u_{2}} \frac{\left\|u_{1}-u_{2}\right\|_{X}}{\left\|f\left(u_{1}\right)-f\left(u_{2}\right)\right\|_{X}}=\sup _{v_{1} \neq v_{2}} \frac{\left\|f^{-1}\left(v_{1}\right)-f^{-1}\left(v_{2}\right)\right\|_{X}}{\left\|v_{1}-v_{2}\right\|_{X}}=L_{X}\left[f^{-1}\right] .
$$

Lemma 2.5 together with the inequality $m_{X}[f] \leq l_{X}[f]$ yields the following corollary.

Corollary 2.6. If $m_{X}[f]>0$, then $f$ is injective and $L_{X}\left[f^{-1}\right] \leq m_{X}[f]^{-1}$. 


\section{Direct product SPACES}

Introduce the Hilbert space $X^{s}$, i.e., the direct product of $s$ spaces $X$, equipped with the inner product $\langle\cdot, \cdot\rangle_{D, X}$ and the corresponding norm $\|\cdot\|_{D, X}$. Elements $U \in X^{s}$ are denoted as $U=\left(U_{1}, \ldots, U_{s}\right)^{\mathrm{T}}$ with $U_{i} \in X$, and the inner product is defined by

$$
\langle U, V\rangle_{D, X}:=\sum_{i=1}^{s} d_{i}\left\langle U_{i}, V_{i}\right\rangle_{X}
$$

where $D=\operatorname{diag}\left(d_{1}, \ldots, d_{s}\right)$ and $d_{i}>0$ for $i=1, \ldots, s$. Note that we drop the subscript $X$ in $\langle\cdot, \cdot\rangle_{D, X}$ when $X=\mathbb{R}$ in order to simplify the notation. The error analysis requires only two types of maps on $X^{s}$ : to every map $f$ on $X$ relate the map $\mathcal{F}: D(f)^{s} \rightarrow X^{s}$ defined as $\mathcal{F}(U)_{i}:=f\left(U_{i}\right)$ for $i=1, \ldots, s$. Furthermore, to every real matrix $A=\left\{a_{i j}\right\}_{i, j=1}^{s}$ relate the linear map $\mathcal{A}: X^{s} \rightarrow X^{s}$ defined as

$$
(\mathcal{A} U)_{i}:=\sum_{j=1}^{s} a_{i j} U_{j} \quad \text { for } i=1, \ldots, s .
$$

Relating the logarithmic Lipschitz constants of $\mathcal{F}$ to the constants of $f$, as presented in the lemma below, follows trivially from the definition of $\langle\cdot, \cdot\rangle_{D, X}$.

Lemma 3.1. $M_{D, X}[\mathcal{F}]=M_{X}[f]$ and $m_{D, X}[\mathcal{F}]=m_{X}[f]$.

Similar relations hold between the logarithmic Lipschitz constants of $\mathcal{A}$ and $A$.

Lemma 3.2. $M_{D, l_{2}}[\mathcal{A}]=M_{D}[A]$ and $m_{D, l_{2}}[\mathcal{A}]=m_{D}[A]$.

Proof. It is sufficient to prove the first equality. If the elements of $U \in l_{2}^{s}$ are denoted as $U_{i}=\left(U_{i}^{1}, U_{i}^{2}, \ldots\right) \in l_{2}$, then

$$
\langle U, \mathcal{A} U\rangle_{D, l_{2}}=\sum_{i=1}^{s} d_{i} \sum_{k=1}^{\infty} U_{i}^{k} \sum_{j=1}^{s} a_{i j} U_{j}^{k}=\sum_{k=1}^{\infty} \sum_{i=1}^{s} d_{i} U_{i}^{k} \sum_{j=1}^{s} a_{i j} U_{j}^{k} \leq M_{D}[A]\|U\|_{D, l_{2}}^{2} .
$$

Hence, $M_{D, l_{2}}[\mathcal{A}] \leq M_{D}[A]$, and as

$$
M_{D, l_{2}}[\mathcal{A}] \geq \sup _{\left\{U \in l_{2}^{s} \backslash 0 \mid U_{i}=\left(U_{i}^{1}, 0,0, \ldots\right)\right\}} \frac{\langle U, \mathcal{A} U\rangle_{D, l_{2}}}{\|U\|_{D, l_{2}}^{2}}=M_{D}[A],
$$

the equality holds.

Corollary 3.3. If $X$ is a separable (infinite-dimensional) Hilbert space, then $M_{D, X}[\mathcal{A}]=M_{D}[A]$ and $m_{D, X}[\mathcal{A}]=m_{D}[A]$.

Proof. As $X$ is separable, there exists a linear bijection $\phi: X \rightarrow l_{2}$ such that $\langle u, v\rangle_{X}=\langle\phi u, \phi v\rangle_{l_{2}}$. Due to the linearity of $\phi$,

$$
\langle U, \mathcal{A} U\rangle_{D, X}=\sum_{i=1}^{s} d_{i}\left\langle\phi U_{i}, \phi \sum_{j=1}^{s} a_{i j} U_{j}\right\rangle_{l_{2}}=\sum_{i=1}^{s} d_{i}\left\langle\phi U_{i}, \sum_{j=1}^{s} a_{i j} \phi U_{j}\right\rangle_{l_{2}}=\langle V, \mathcal{A} V\rangle_{D, l_{2}}
$$

where $V_{i}:=\phi U_{i}$. The desired equalities are now obtained, as $\phi$ is a bijection.

Naturally, Corollary 3.3 is also valid for finite-dimensional Hilbert spaces, which follows by replacing $l_{2}$ by $\left(\mathbb{R}^{\operatorname{dim} X},\|\cdot\|_{l_{2}}\right.$ ) in the proofs. The same type of results as in Lemma 3.1 and Corollary 3.3 can also be derived for the Lipschitz constants, but they are omitted, as $f$ may have $L_{X}[f]=\infty$ and $L_{D, X}[\mathcal{A}]$ is obviously bounded for all $s$. 


\section{Problem Setting}

Let $X$ be a real-valued separable Hilbert space and consider the nonlinear evolution equation

$$
\dot{u}=f(u), \quad u(0)=\eta \in D(f),
$$

where $u:[0, \infty) \rightarrow D(f)$ and the vector field $f$ is a nonlinear map on $X$ with $M_{X}[f] \leq 0$ and $R(I-h f)=X$ for all $h>0$. Such vector fields are usually referred to as $m$-dissipative [1].

Definition 4.1. A function $u:[0, \infty) \rightarrow D(f)$ is said to be a strong solution of equation (4.1) on $[0, \infty)$ if $u(0)=\eta$ and $\dot{u}=f(u)$ a.e. on $(0, \infty)$.

Proposition 4.2. The function $u: t \mapsto \mathrm{e}^{t f}(\eta)$ is the unique strong solution of equation (4.1), where $\mathrm{e}^{\text {tf }}$ is a nonlinear, nonexpansive semigroup on $D(f)$ defined as

$$
\mathrm{e}^{t f}(\eta):=\lim _{n \rightarrow \infty}\left(I-\frac{t}{n} f\right)^{-n}(\eta) .
$$

See, for example, Theorem 31.A and Corollary 31.1 in 17] for the proof and further properties of the semigroup $\mathrm{e}^{t f}$. Extensions of these results to Banach spaces are treated in [1, 2].

Example 4.3. Let $\Omega$ be a bounded region in $\mathbb{R}^{m}$ and define the evolution triple $\left(V, X, V^{*}\right)$ by $V \subset X=X^{*} \subset V^{*}$, where $X:=L_{2}(\Omega)$ and $V:=W_{0}^{1, p}(\Omega)$ with $2 \leq p<\infty$. Consider the map $\Delta_{p}: C_{0}^{\infty}(\Omega) \rightarrow X$ defined as

$$
\Delta_{p}: u \mapsto \sum_{i=1}^{m} \partial_{i}\left(\left|\partial_{i} u\right|^{p-2} \partial_{i} u\right),
$$

and its energetic extension $\Delta_{E, p}: V \rightarrow V^{*}$, i.e.,

$$
\Delta_{E, p}: u \mapsto-\int_{\Omega} \sum_{i=1}^{m}\left(\left|\partial_{i} u\right|^{p-2} \partial_{i} u\right) \partial_{i}(\cdot) \mathrm{d} x .
$$

Then, the map $f: D \rightarrow X$, with $D:=\Delta_{E, p}^{-1}\left(X^{*}\right)$ and $\langle f(u), \cdot\rangle_{X}=\Delta_{E, p}(u)$ for all $u \in D$, fits into our framework; see Sections 26.5 and 31.5 in [17] for details and generalizations.

The Runge-Kutta approximation $u_{n+1} \in X$ of $u\left(t_{n+1}\right)$ for $n \geq 0$ is defined by the difference equation

$$
\left\{\begin{array}{l}
U_{i}=u_{n}+h \sum_{j=1}^{s} a_{i j} f\left(U_{j}\right), \quad i=1, \ldots, s \\
u_{n+1}=u_{n}+h \sum_{i=1}^{s} b_{i} f\left(U_{i}\right)
\end{array}\right.
$$

where $u_{0}:=\eta$ and $t_{n}:=n h$. To every Runge-Kutta method we relate the vectors $b:=\left(b_{1}, \ldots, b_{s}\right)^{\mathrm{T}}$ and $c:=\left(c_{1}, \ldots, c_{s}\right)^{\mathrm{T}}$ together with the matrix $A:=\left\{a_{i j}\right\}_{i, j=1}^{s}$. In the error analysis below the following consistency (C1), (C2), and stability (S) assumptions are needed:

(C1) The analytic solution $u \in C^{q+1}([0, \infty), X)$ satisfies $\dot{u}=f(u)$ everywhere on $[0, \infty)$. 
(C2) The coefficients of the Runge-Kutta method satisfy the algebraic relations (order conditions)

$$
\sum_{i=1}^{s} b_{i} c_{i}^{k-1}=1 / k \quad \text { and } \quad \sum_{j=1}^{s} a_{i j} c_{j}^{k-1}=c_{i}^{k} / k \quad \text { for } k=1, \ldots, q .
$$

(S) The matrix $S:=\left\{b_{i} a_{i j}+b_{j} a_{j i}-b_{i} b_{j}\right\}_{i, j=1}^{s}$ has $m_{I}[S] \geq 0$ and the coefficients $b_{i} \geq 0$ for $i=1, \ldots, s$.

Assumption (S) is usually referred to as algebraic stability [6]. Next, for every $n \geq 0$, introduce the local stage residuals $L_{i} \in X$ and the local residual $l \in X$ defined as

$$
\left\{\begin{array}{l}
L_{i}:=u\left(t_{n}+c_{i} h\right)-u\left(t_{n}\right)-h \sum_{j=1}^{s} a_{i j} f\left(u\left(t_{n}+c_{j} h\right)\right), \quad i=1, \ldots, s, \\
l:=u\left(t_{n+1}\right)-u\left(t_{n}\right)-h \sum_{i=1}^{s} b_{i} f\left(u\left(t_{n}+c_{i} h\right)\right) .
\end{array}\right.
$$

Theorem 4.4. If assumptions (C1) and (C2) hold, then $\left\|L_{i}\right\|_{X}$ and $\|l\|_{X}$ are $\mathrm{O}\left(h^{q+1}\right)$.

Proof. Assumption (C1) implies that $f\left(u\left(t_{n}+c_{i} h\right)\right)$ can be written as a Taylor expansion of order $q$, since integration by parts is possible in the context of Bochner integrals. Thus, the proof follows by a Taylor expansion of $L_{i}$ and $l$, where the terms of order less than or equal to $q$ cancel out, due to assumption (C2).

Example 4.5. Assumption (C1) is for example valid if $f$ is linear and $\eta \in D\left(f^{q+1}\right)$; see [13. The Gauss, Radau I/IIA, and Lobatto IIIC methods all satisfy assumptions (C2) and (S); see [3, 6].

\section{EXISTENCE OF A UNIQUE APPROXIMATION}

Introduce the nonlinear map $\mathcal{F}: D(f)^{s} \rightarrow X^{s}$ related to the vector field $f$ and the linear map $\mathcal{A}: X^{s} \rightarrow X^{s}$ related to the matrix $A$. Then equation (4.2) can be written as

$$
\left\{\begin{array}{l}
(\mathcal{I}-h \mathcal{A F})(U)=Y \\
u_{n+1}=u_{n}+h \sum_{i=1}^{s} b_{i} f\left(U_{i}\right)
\end{array}\right.
$$

where $U:=\left(U_{1}, \ldots, U_{s}\right)^{\mathrm{T}}$ and $Y:=\left(u_{n}, \ldots, u_{n}\right)^{\mathrm{T}}$. Thus, proving that there exists a unique solution of (4.2) is reduced to proving that the map $\mathcal{I}-h \mathcal{A F}: D(f)^{s} \rightarrow X^{s}$ is a bijection.

Lemma 5.1. If $\mathcal{E}: X^{s} \rightarrow X^{s}$ and $\mu \in\left(0, L_{D, X}[\mathcal{E}]^{-1}\right)$, then $\mathcal{I}+\mu(\mathcal{E}-h \mathcal{F})$ : $D(f)^{s} \rightarrow X^{s}$ is a bijection for all $h>0$.

Proof. By the hypotheses imposed on $f, m_{D, X}[\mathcal{I}-h \mathcal{F}] \geq 1-h M_{X}[f] \geq 1$ and $R(\mathcal{I}-h \mathcal{F})=X^{s}$ for all $h>0$. Corollary 2.6 therefore implies that $(\mathcal{I}-h \mathcal{F})^{-1}$ : $X^{s} \rightarrow D(f)^{s}$ is well defined and

$$
L_{D, X}\left[(\mathcal{I}-h \mathcal{F})^{-1}\right] \leq\left(1-h M_{X}[f]\right)^{-1} \leq 1
$$

for all $h>0$. Thus, for all $Y \in X^{s}$ and $\mu>0$

$$
(\mathcal{I}+\mu(\mathcal{E}-h \mathcal{F}))(U)=Y \quad \Leftrightarrow \quad U=(\mathcal{I}-\mu h \mathcal{F})^{-1}(Y-\mu \mathcal{E}(U))
$$


TABLE 1. Optimal $D$-matrices for a selection of Runge-Kutta methods.

\begin{tabular}{l|c|l} 
Method & $D$ & $m_{D}\left[A^{-1}\right]$ \\
\hline Gauss & $B\left(C^{-1}-I\right)$ & $>0$ for all $s$ \\
Radau IA & $B(I-C)$ & $>0$ for all $s$ \\
Radau IIA & $B C^{-1}$ & $>0$ for all $s$ \\
Lobatto IIIC & $B$ & $>0$ for $s=2$ and \\
& & $=0$ for $s \geq 3$ \\
\hline
\end{tabular}

and $L_{D, X}\left[(\mathcal{I}-\mu h \mathcal{F})^{-1}(Y-\mu \mathcal{E}(\cdot))\right]<1$ when $\mu<L_{D, X}[\mathcal{E}]^{-1}$. Banach's fixed-point theorem now yields that for every $Y \in X^{s}$ there exists a unique $U \in D(f)^{s}$ such that $(\mathcal{I}+\mu(\mathcal{E}-h \mathcal{F}))(U)=Y$.

Theorem 5.2. If $A$ is invertible and there exists a positive diagonal matrix $D$ such that $m_{D}\left[A^{-1}\right]-h M_{X}[f]>0$ for all $h>0$, then $\mathcal{I}-h \mathcal{A F}: D(f)^{s} \rightarrow X^{s}$ is a bijection.

Proof. Let $Y \in X^{s}$. Then

$$
(\mathcal{I}-h \mathcal{A} \mathcal{F})(U)=Y \quad \Leftrightarrow \quad\left(\mathcal{I}+\mu\left(\mathcal{A}^{-1}-h \mathcal{F}\right)\right)(U)=\mu \mathcal{A}^{-1} Y+U .
$$

By Lemma 5.1 with $\mathcal{E}=\mathcal{A}^{-1}$ and $\mu \in\left(0, L_{D, X}\left[\mathcal{A}^{-1}\right]^{-1}\right)$, the map $\mathcal{I}+\mu\left(\mathcal{A}^{-1}-h \mathcal{F}\right)$ : $D(f)^{s} \rightarrow X^{s}$ is a bijection. Furthermore,

$$
m_{D, X}\left[\mathcal{I}+\mu\left(\mathcal{A}^{-1}-h \mathcal{F}\right)\right] \geq 1+\mu\left(m_{D}\left[A^{-1}\right]-h M_{X}[f]\right)>1,
$$

and Corollary 2.6 gives that $L_{D, X}\left[\left(\mathcal{I}+\mu\left(\mathcal{A}^{-1}-h \mathcal{F}\right)\right)^{-1}\right]<1$. Hence, the map

$$
U \mapsto\left(\mathcal{I}+\mu\left(\mathcal{A}^{-1}-h \mathcal{F}\right)\right)^{-1}(W+U)
$$

is a contraction on $X^{s}$ for all $W \in X^{s}$, and the proof follows again by Banach's fixed-point theorem.

Example 5.3. The hypotheses imposed on the matrix $A$ in Theorem 5.2 are fulfilled for the Gauss, Radau I/IIA, and Lobatto IIIC methods with the matrix $D$ specified in Table 1, where $B:=\operatorname{diag}\left(b_{1}, \ldots, b_{s}\right)$ and $C:=\operatorname{diag}\left(c_{1}, \ldots, c_{s}\right)$; see the proof of Theorem 14.5 in [6].

\section{Global ERror anAlysis}

For every $n \geq 0$ define $v$ as one Runge-Kutta step starting from the analytic solution, i.e.,

$$
\left\{\begin{array}{l}
V_{i}=u\left(t_{n}\right)+h \sum_{j=1}^{s} a_{i j} f\left(V_{j}\right), \quad i=1, \ldots, s \\
v=u\left(t_{n}\right)+h \sum_{i=1}^{s} b_{i} f\left(V_{i}\right) .
\end{array}\right.
$$

The global error $e_{n+1}:=u\left(t_{n+1}\right)-u_{n+1}$ can then be split into

$$
e_{n+1}=u\left(t_{n+1}\right)-v+v-u_{n+1},
$$

and deriving bounds of the term $u\left(t_{n+1}\right)-v$ are related to the consistency of the method, whereas the term $v-u_{n+1}$ can be bounded if the method is stable. 
Theorem 6.1. If assumptions $(\mathrm{C} 1)$ and $(\mathrm{C} 2)$ hold, $A$ is invertible, and there exists a positive diagonal matrix $D$ such that $m_{D}\left[A^{-1}\right]-h M_{X}[f]>0$ for all $h>0$, then

$$
\left\|u\left(t_{n+1}\right)-v\right\|_{X} \leq C\left(1+\frac{L_{D, X}\left[\mathcal{A}^{-1}\right]}{m_{D}\left[A^{-1}\right]-h M_{X}[f]}\right) h^{q+1},
$$

where the positive constant $C$ is independent of $n, m_{D}\left[A^{-1}\right]$, and $h M_{X}[f]$.

Proof. Define the vectors $Z:=\left(u\left(t_{n}+h c_{1}\right), \ldots, u\left(t_{n}+h c_{s}\right)\right)^{\mathrm{T}}, V:=\left(V_{1}, \ldots, V_{s}\right)^{\mathrm{T}}$, and $L:=\left(L_{1}, \ldots, L_{s}\right)^{\mathrm{T}}$. Then equations (4.3) and (6.1) yield the relations

$$
\left\{\begin{array}{l}
L=(\mathcal{I}-h \mathcal{A F})(Z)-(\mathcal{I}-h \mathcal{A} \mathcal{F})(V), \\
u\left(t_{n+1}\right)-v=h \sum_{i=1}^{s} b_{i}\left(f\left(Z_{i}\right)-f\left(V_{i}\right)\right)+l,
\end{array}\right.
$$

where $\left\|L_{i}\right\|_{X}$ and $\|l\|_{X}$ are of $\mathrm{O}\left(h^{q+1}\right)$ by Theorem 4.4. Thus,

$$
\left\|u\left(t_{n+1}\right)-v\right\|_{X} \leq\left(s \max _{1 \leq i \leq s}\left|b_{i}\right|\right) \max _{1 \leq i \leq s} h\left\|f\left(Z_{i}\right)-f\left(V_{i}\right)\right\|_{X}+\|l\|_{X} .
$$

The proof now follows if the term $\max _{i} h\left\|f\left(Z_{i}\right)-f\left(V_{i}\right)\right\|_{X}$ is bounded by $\max _{i}\left\|L_{i}\right\|_{X}$. To this end, apply the functional $\left\|\mathcal{A}^{-1}(\cdot)\right\|_{D, X}$ to the first equation of (6.2) and observe that

$$
l_{D, X}\left[\mathcal{A}^{-1}-h \mathcal{F}\right] \geq m_{D, X}\left[\mathcal{A}^{-1}-h \mathcal{F}\right] \geq m_{D}\left[A^{-1}\right]-h M_{X}[f]>0,
$$

which implies the inequality

$$
\|Z-V\|_{D, X} \leq \frac{L_{D, X}\left[\mathcal{A}^{-1}\right]}{m_{D}\left[A^{-1}\right]-h M_{X}[f]}\|L\|_{D, X}
$$

Furthermore, as $h(\mathcal{F}(Z)-\mathcal{F}(V))=\mathcal{A}^{-1}(Z-V-L)$, one also obtains the inequality

$$
h\|\mathcal{F}(Z)-\mathcal{F}(V)\|_{D, X} \leq L_{D, X}\left[\mathcal{A}^{-1}\right]\left(1+\frac{L_{D, X}\left[\mathcal{A}^{-1}\right]}{m_{D}\left[A^{-1}\right]-h M_{X}[f]}\right)\|L\|_{D, X} .
$$

The desired bound is now obtained, as the norms $U \mapsto \max _{1 \leq i \leq s}\left\|U_{i}\right\|_{X}$ and $\|\cdot\|_{D, X}$ are equivalent on $X^{s}$.

Theorem 6.2. If assumption (S) holds, i.e., $b_{i} \geq 0$ and $m_{I}[S] \geq 0$, then

$$
\left\|v-u_{n+1}\right\|_{X} \leq\left\|e_{n}\right\|_{X} .
$$

Proof. Subtracting equation (4.2) from (6.1) gives

$$
\left\{\begin{array}{l}
E=(\mathcal{I}-h \mathcal{A} \mathcal{F})(V)-(\mathcal{I}-h \mathcal{A} \mathcal{F})(U), \\
v-u_{n+1}=e_{n}+h \sum_{i=1}^{s} b_{i}\left(f\left(V_{i}\right)-f\left(U_{i}\right)\right),
\end{array}\right.
$$

where $E:=\left(e_{n}, \ldots, e_{n}\right)^{\mathrm{T}}$. For compact notation, define $\Delta U:=V-U$ and $\Delta \mathcal{F}:=\mathcal{F}(V)-\mathcal{F}(U)$. Then

$$
\begin{aligned}
& \left\|v-u_{n+1}\right\|_{X}^{2}=\left\langle e_{n}+h \sum_{i=1}^{s} b_{i} \Delta \mathcal{F}_{i}, e_{n}+h \sum_{j=1}^{s} b_{j} \Delta \mathcal{F}_{j}\right\rangle_{X} \\
& =\left\|e_{n}\right\|_{X}^{2}+2 h \sum_{i=1}^{s} b_{i}\left\langle E_{i}, \Delta \mathcal{F}_{i}\right\rangle_{X}+h^{2} \sum_{i, j=1}^{s} b_{i} b_{j}\left\langle\Delta \mathcal{F}_{i}, \Delta \mathcal{F}_{j}\right\rangle_{X} \\
& =\left\|e_{n}\right\|_{X}^{2}+2 h \sum_{i=1}^{s} b_{i}\left\langle\Delta U_{i}, \Delta \mathcal{F}_{i}\right\rangle_{X}-h^{2} \sum_{i, j=1}^{s}\left(b_{i} a_{i j}+b_{j} a_{j i}-b_{i} b_{j}\right)\left\langle\Delta \mathcal{F}_{i}, \Delta \mathcal{F}_{j}\right\rangle_{X} .
\end{aligned}
$$


Let $\mathcal{S}: X^{s} \rightarrow X^{s}$ be the linear map related to the matrix $S$. Thus, by the definition of $\langle\cdot, \cdot\rangle_{D, X}$,

$$
\sum_{i, j=1}^{s}\left(b_{i} a_{i j}+b_{j} a_{j i}-b_{i} b_{j}\right)\left\langle\Delta \mathcal{F}_{i}, \Delta \mathcal{F}_{j}\right\rangle_{X}=\langle\Delta \mathcal{F}, \mathcal{S} \Delta \mathcal{F}\rangle_{I, X} .
$$

The proof now follows as $b_{i} M_{X}[f] \leq 0$, and $m_{I, X}[\mathcal{S}]=m_{I}[S] \geq 0$.

Finally, by combining Theorems 6.1 and 6.2 , one obtains the convergence results below.

Corollary 6.3. If the hypotheses of Theorems 6.1 and 6.2 are fulfilled, then

$$
\left\|e_{n}\right\|_{X} \leq C t_{n}\left(1+\frac{L_{D, X}\left[\mathcal{A}^{-1}\right]}{m_{D}\left[A^{-1}\right]-h M_{X}[f]}\right) h^{q} .
$$

Proof. By the derived consistency and stability bounds, we have

$$
\left\|e_{n+1}\right\|_{X} \leq\left\|e_{n}\right\|_{X}+C\left(1+\frac{L_{D, X}\left[\mathcal{A}^{-1}\right]}{m_{D}\left[A^{-1}\right]-h M_{X}[f]}\right) h^{q+1} .
$$

As $e_{0}=0$, the proof now follows by an $n$-fold repetition of the inequality above.

Note that the convergence orders derived in Corollary 6.3, i.e.,

$$
\left\|e_{n}\right\|_{X}= \begin{cases}\mathrm{O}\left(h^{q}\right) & \text { if } m_{D}\left[A^{-1}\right]>0 \text { and } M_{X}[f] \leq 0 \\ \mathrm{O}\left(h^{q-1}\right) & \text { if } m_{D}\left[A^{-1}\right]=0 \text { and } M_{X}[f]<0\end{cases}
$$

are the same as the ones obtained when applying the $B$-convergence theory to Runge-Kutta approximations of ordinary differential equations; see Theorem 15.3 in [6].

\section{Generalization of the ERror ANALYSis}

The error analysis can be generalized to vector fields satisfying the conditions

$$
M_{X}[f]<\infty \quad \text { and } \quad R(I-h f)=X \quad \forall h>0 \text { s.t. } h M_{X}[f]<1 .
$$

Such vector fields can be interpreted as maps which can be shifted to $m$-dissipative maps, i.e., $f-M_{X}[f] I$ is $m$-dissipative. The analytic solution of the related evolution equation is still given by the function $u: t \mapsto \mathrm{e}^{t f}(\eta)$, where the nonlinear semigroup $\mathrm{e}^{t f}$, defined in Proposition 4.2, satisfies

$$
L_{X}\left[\mathrm{e}^{t f}\right] \leq \mathrm{e}^{t M_{X}[f]}
$$

see [1, 2] for proofs and further results. As the error analysis has already been made for $m$-dissipative maps, we now consider vector fields and stepsizes satisfying $M_{X}[f] \in(0, \infty)$ and $1-h M_{X}[f]>0$. Proving that there exists a unique RungeKutta approximation again follows if the map $\mathcal{I}-h \mathcal{A F}: D(f)^{s} \rightarrow X^{s}$ is a bijection.

Lemma 7.1. If $\mathcal{E}: X^{s} \rightarrow X^{s}$ and $\mu \in\left(0,\left(L_{D, X}[\mathcal{E}]+h M_{X}[f]\right)^{-1}\right)$, then $\mathcal{I}+$ $\mu(\mathcal{E}-h \mathcal{F}): D(f)^{s} \rightarrow X^{s}$ is a bijection for all $h>0$.

Proof. As $\mu h M_{X}[f]<1$, Corollary 2.6 yields that $(\mathcal{I}-\mu h \mathcal{F})^{-1}: X^{s} \rightarrow D(f)^{s}$ is well defined and

$$
L_{D, X}\left[(\mathcal{I}-\mu h \mathcal{F})^{-1}(Y-\mu \mathcal{E}(\cdot))\right] \leq \mu L_{D, X}[\mathcal{E}]\left(1-\mu h M_{X}[f]\right)^{-1}<1,
$$

when $\mu<\left(L_{D, X}[\mathcal{E}]+h M_{X}[f]\right)^{-1}$. The rest of the proof follows as in Lemma 5.1 . 
Lemma 7.1 and a $\mu$ in the interval $\left(0,\left(L_{D, X}\left[\mathcal{A}^{-1}\right]+h M_{X}[f]\right)^{-1}\right)$ imply that the proof of Theorem 5.2 is valid in the present context, which yields that the map $\mathcal{I}-h \mathcal{A F}$ is a bijection for all $h>0$ such that $m_{D}\left[A^{-1}\right]-h M_{X}[f]>0$. Furthermore, just as $\mathrm{e}^{t f}$ is no longer nonexpansive when $M_{X}[f]>0$, the stability properties of the Runge-Kutta approximation are weakened.

Theorem 7.2. If $m_{D}\left[A^{-1}\right]-h M_{X}[f]>0$ and assumption (S) holds, then

$$
\left\|v-u_{n+1}\right\|_{X} \leq\left(1+C_{0} h\right)\left\|e_{n}\right\|_{X},
$$

where the positive constant $C_{0}$ is independent of $n$.

Proof. The proof of Theorem 6.2 gives the relation

$$
E=(\mathcal{I}-h \mathcal{A F})(V)-(\mathcal{I}-h \mathcal{A F})(U)
$$

and, as $M_{X}[f]>0$, the inequality

$$
\left\|v-u_{n+1}\right\|_{X}^{2} \leq\left\|e_{n}\right\|_{X}^{2}+2 h M_{X}[f]\left(s \max _{1 \leq i \leq s} b_{i}\right) \max _{1 \leq i \leq s}\left\|V_{i}-U_{i}\right\|_{X}^{2} .
$$

The same technique as in Theorem 6.1 may be used, i.e., applying the functional $\left\|\mathcal{A}^{-1}(\cdot)\right\|_{D, X}$ to (7.1) and observing that $l_{D, X}\left[\mathcal{A}^{-1}-h \mathcal{F}\right] \geq m_{D}\left[A^{-1}\right]-h M_{X}[f]$, which implies the bound

$$
\|V-U\|_{D, X} \leq \frac{L_{D, X}\left[\mathcal{A}^{-1}\right]}{m_{D}\left[A^{-1}\right]-h M_{X}[f]}\|E\|_{D, X} .
$$

The desired stability result follows as the norms $U \mapsto \max _{1 \leq i \leq s}\left\|U_{i}\right\|_{X}$ and $\|\cdot\|_{D, X}$ are equivalent on $X^{s}$ and $\sqrt{1+x} \leq 1+x$ for $x \geq 0$.

In conclusion, for $M_{X}[f] \in(0, \infty)$ the error analysis requires a stepsize restriction $h \in\left(0, h_{0}\right]$, where

$$
\min \left\{1, m_{D}\left[A^{-1}\right]\right\}-h_{0} M_{X}[f]>0,
$$

and one has a global error bound as presented below.

Corollary 7.3. If $h \in\left(0, h_{0}\right]$ and the hypotheses of Theorems 6.1 and 7.2 are fulfilled, then

$$
\left\|e_{n}\right\|_{X} \leq C \frac{\mathrm{e}^{C_{0} t_{n}}-1}{C_{0}}\left(1+\frac{L_{D, X}\left[\mathcal{A}^{-1}\right]}{m_{D}\left[A^{-1}\right]-h M_{X}[f]}\right) h^{q} .
$$

Proof. If $h \in\left(0, h_{0}\right]$, then the proof of Theorem 6.1 is valid for $M_{X}[f] \in(0, \infty)$, which together with Theorem 7.2 yields the bound

$$
\left\|e_{n+1}\right\|_{X} \leq\left(1+C_{0} h\right)\left\|e_{n}\right\|_{X}+C\left(1+\frac{L_{D, X}\left[\mathcal{A}^{-1}\right]}{m_{D}\left[A^{-1}\right]-h M_{X}[f]}\right) h^{q+1} .
$$

As $e_{0}=0$, the proof now follows by an $n$-fold repetition of the inequality above, together with the observation that $\sum_{i=0}^{n}\left(1+C_{0} h\right)^{i} \leq\left(\mathrm{e}^{C_{0}(n+1) h}-1\right) / C_{0} h$.

\section{Conclusions}

Global error bounds are derived for Runge-Kutta time discretizations of fully nonlinear evolution equations governed by $m$-dissipative vector fields on Hilbert spaces. The analysis is carried out using the theory of logarithmic Lipschitz constants, which enable us to extend the classical $B$-convergence theory of ordinary differential equations to our infinite-dimensional setting. The convergence orders derived here are the same as the ones obtained when applying the $B$-convergence 
theory to approximations of ordinary differential equations, i.e., algebraically stable Runge-Kutta methods with stage order $q$ have a convergence order of at least $q-1$ or $q$, depending on the monotonicity properties of the methods.

\section{ACKNOWLEDGMENTS}

The author would like to thank Gustaf Söderlind and Alexander Ostermann for all the inspiring discussions.

\section{REFERENCES}

1. V. Barbu, Nonlinear semigroups and differential equations in Banach spaces, Noordhoff International Publishing, Leyden, 1976.

2. M. G. Crandall, Nonlinear semigroups and evolution governed by accretive operators, Proc. Sympos. Pure Math. 45 (1986), pp. 305-337.

3. K. Dekker and J. G. Verwer, Stability of Runge-Kutta methods for stiff nonlinear differential equations, North-Holland Publishing Co., Amsterdam, 1984.

4. R. Frank, J. Schneid and C. W. Ueberhuber, Order results for implicit Runge-Kutta methods applied to stiff systems, SIAM J. Numer. Anal. 22 (1985), pp. 515-534.

5. C. González, A. Ostermann, C. Palencia and M. Thalhammer, Backward Euler discretization of fully nonlinear parabolic problems, Math. Comp. 71 (2002), pp. 125-145.

6. E. Hairer and G. Wanner, Solving ordinary differential equations. II. Stiff and differentialalgebraic problems, Second edition, Springer-Verlag, Berlin, 1996.

7. E. Hansen, Convergence of multistep time discretizations of nonlinear dissipative evolution equations, To appear in SIAM J. Numer. Anal.

8. I. Higueras and G. Söderlind, Logarithmic norms and nonlinear DAE stability, BIT 42 (2002), pp. 823-841.

9. Ch. Lubich and A. Ostermann, Runge-Kutta methods for parabolic equations and convolution quadrature, Math. Comp. 60 (1993), pp. 105-131.

10. (1995), pp. 601-627.

11. A. Ostermann and M. Thalhammer, Convergence of Runge-Kutta methods for nonlinear parabolic equations, Appl. Numer. Math. 42 (2002), pp. 367-380.

12. A. Ostermann, M. Thalhammer and G. Kirlinger, Stability of linear multistep methods and applications to nonlinear parabolic problems, Appl. Numer. Math. 48 (2004), pp. 389-407.

13. A. Pazy, Semigroups of linear operators and applications to partial differential equations, Springer-Verlag, New York, 1983.

14. G. Söderlind, On nonlinear difference and differential equations, BIT 24 (1984), pp. 667-680.

15. - Bounds on nonlinear operators in finite-dimensional Banach spaces, Numer. Math. 50 (1986), pp. 27-44.

16. V. Thomée, Galerkin finite element methods for parabolic problems, Springer-Verlag, New York, 1997.

17. E. Zeidler, Nonlinear functional analysis and its applications. II/B. Nonlinear monotone operators, Springer-Verlag, New York, 1990.

Centre for Mathematical Sciences, Lund University, Box 118, SE-221 00 Lund, SweDEN

E-mail address: eskil@maths.lth.se 